\title{
Creative Commonsense? An analysis of tensions between Copyright Law and Creative
}

\section{Commons}

Dr Nick Scharf 1

\section{Legal context}

The Creative Commons (CC) movement operates as an important counterpoint to the expansionist copyright regime in the digital environment relating to recorded music by seeking to create a free and simple licensing mechanism that operates to construct a pool of content that creators can use without charge. It creates an alternative model for creators to use in order to authorise the use/reuse of their work that circumvents the traditional market for copyright content as well as fostering its own CC-orientated intermediary outlets.

\section{Key points}

$\mathrm{CC}$ depends on copyright's underlying proprietary system. However, CC arguably constrains creators in terms of the content they can use to produce new works and does nothing to necessarily overcome the complexities posed by copyright infringement should a licence be breached. Nonetheless, there are a number of intermediaries that provide CC-licenced content which suggests that the movement has traction. Following an analysis of the CCorientated intermediaries in comparison with those engendered by copyright, it may be argued that the $\mathrm{CC}$ licensing system makes it difficult to transition from an amateur to a professional context. Nonetheless, its symbolic value remains important.

\footnotetext{
1 Lecturer in Intellectual Property Law, University of East Anglia (UEA) Law School
} 


\section{Practical significance}

This piece will address the intermediary market structure for CC works as well as the potential difficulties commercial exploitation in order to evaluate whether or not it can be seen as a beneficial system for content creation and dissemination. As is CC inherently limited by the intermediary structure built upon 'free' use, this may hinder a transition to revenue-generating creative activity. 


\section{Introduction}

From a creator and user perspective, the loss of faith in copyright law, and its enforcement has led to a significant reappraisal of the role of private law in the digital environment2. One initiative which warrants consideration in this case, is Creative Commons (CC) which stands as a positive3 counterpoint, to the preceding expansionist nature of copyright law. The movement was inspired by what was perceived as a threat to culture as a result of the influence of copyright law on creativity4. As a counter-point to this, $\mathrm{CC}$ aims to '...build a more equitable, accessible, and innovative world'.5 Its premise is to relocate power from rightsholders to creators, who are afforded options to govern how their works may be used and re-used. It also has the benefit of recognising that in the digital environment, the division between authors and users may be indistinct such that now users have the power and tools at their disposal to engage in creating their own content. As such, this piece uses the collective term 'creators' to refer to both parties.

It will explore the relationship between $\mathrm{CC}$ and copyright to demonstrate that although $\mathrm{CC}$ is dependent on the copyright system as a source of legal rights which can be forgone through the licensing options, its aim to create alternative source(s) of content circumvent the traditional market for copyright content. As such, a divergence becomes evident between the markets for CC-licensed content and that of copyrighted content. Although the $\mathrm{CC}$ vision realises the full potential of the Internet for content sharing and reuse, the commercial realities of the online intermediary landscape suggest that the movement

\footnotetext{
2 K Bowery, Law \& Internet Cultures (Cambridge University Press 2005) 164

3 S Corbett, 'Creative Commons Licences, the Copyright Regime and the Online Community: Is there a Fatal Disconnect?' (2011) MLR 74(4) 503-531, 515

4 Ibid, 507

${ }_{5}$ Creative Commons, 'What we do'<http://creativecommons.org/about $>$ accessed 6 November 2906
} 
may struggle to emerge beyond its relative self-established niche. Whilst the alternative CC offers may be of primary appeal to many creators, the intermediary divergence between CC and copyright may therefore make it difficult for them to transition from an environment of sharing and re-use to one of commercial viability.

The piece begins with a brief overview of the $\mathrm{CC}$ movement and available licences, before examining its relationship with copyright law; concluding that it arguably constrains creators in terms of the content they can use to produce new works and does nothing to necessarily overcome the complexities posed by copyright infringement should a licence be breached. Despite this, there are a number of intermediaries that provide CC-licenced music which suggests that the movement has some traction. However from looking at the market for such works, it will be argued that that it can never form a viable market alternative owing to inherent incompatibility between CC-licenced music the commercial revenue-driven market for music. It will conclude that the $\mathrm{CC}$ licensing system makes it difficult to transition from an amateur to a professional context. Whilst this may be a problem for some (although certainly not all), the music industry engendered by copyright is certainly not free from its own problems broadly caused by a lack of understanding and flexibility toward the very technology that $\mathrm{CC}$ recognises as being crucially important: the Internet. Although there is nothing in copyright law that prevents a creator from exploiting their work and forgoing commercial remuneration, the fact that $\mathrm{CC}$ simply offers an alterative may perhaps be its strongest feature through allowing more formal and indeed symbolic, repositioning of power to creators themselves.

\section{Creative Commons}


Creative Commons (CC) was founded in 2001 by James Boyle, Michael Carroll, Lawrence Lessig, Hal Abelson, Eric Saltzman and Eric Eldred6 who sought an alternative to the traditional copyright system. The CC organisation is a non-profit, US-based establishment which operates as a licensing platform to promote the free use of creative works; both in terms of cost and freedom of use (to a degree)7, which recognises the value arising from the ability to engage and interact with a resource. To an extent, the $\mathrm{CC}$ movement is to be admired as being a positive response to digital copyright infringement by removing restrictions on reproduction and distribution at the source, thus appreciating the normative expectations of users in relation to digital technology8. In contrast, copyright policy in the area has largely been negative (or restrictive) in its response9 and practice.

It assumes that it is possible to replace existing content production and distribution practices 10 with the ultimate objective of positioning creative works as resources available to the public11 as well as 'to promote alternatives to a one-way, passive consumption of

\footnotetext{
${ }_{6}$ Creative Commons, 'History' <http://wiki.creativecommons.org/History> accessed 11 November 2016 7 For Lessig, 'free' translates as the freedom or 'liberty' to use a resource (or content), see L Lessig, The Future of Ideas: the Fate of the Commons in a Connected World (Vintage Books 2001) 12 ${ }_{8}$ See N Scharf, 'Life Through a Lens: A “Lessigan” Model For Understanding Digital Copyright Infringement?’ (2012) Journal of Internet Law 16(1), 18-34

9 M Kriskis and R Petrauskas, 'Lessig’s implications for intellectual property law and beyond them' (2005) International Review of Law, Computers \& Technology 19(3) 305-316, 309 10 N Elkin-Koren, 'What Contracts Cannot Do: The Limits of Private Ordering in Facilitating a Creative Commons' (2005) 74 Fordham Law Review 375-422, 377

11 S Dusollier, 'The Master's Tools v. The Master's House: Creative Commons v. Copyright' (2006) 29 Columbia Journal of Law \& the Arts 3 271-293, 272
} 
commercialized culture'.12 $\mathrm{CC}$ perceives the current copyright regime as a major obstacle for creative activity; something which has been more acutely felt with the development of the Internet and digital technologies. These have created an environment which facilitates the consumption of content which in turn helps to inspire and generate new works; substantially reducing the cost of digital creations and potentially enabling greater participation by users in the creative process13. Crucially, it has also enabled new forms of reuse which were not previously possible from the perspective that copyright law operates too well and is restrictive of such creative practices. This is important as creative practice is generated by and through exposure to other content 14 , and the availability of content is crucial in maintaining a healthy creative environment 15 . Therefore, $\mathrm{CC}$ aims to create and promote an alternative market for the production and consumption of digital content in line with norms and the possibilities afforded by this digital architecture. The overall strategy of the movement can be described as twin-track: a legal component consisting of an author-centric licensing model; and, a symbolic component promoting the philosophies of sharing and contribution 16.

\footnotetext{
12 AK Goss, 'Codifying a Commons: Copyright, Copyleft, and the Creative Commons Project' (2007) 82 ChiKent L Rev 963-996, 977

13 L Lessig, The Future of Ideas: the Fate of the Commons in a Connected World (Vintage Books 2001), 8-9 14 L Lessig, Free Culture: The Nature and Future of Creativity (Penguin Books 2004) 29 and L Lessig, The Future of Ideas: the Fate of the Commons in a Connected World (Vintage Books 2001) 250: “There is no art that doesn't reuse." 15 I Hargreaves, Digital opportunity: a review of intellectual property and growth (Intellectual Property Office 2011), 50 16 S Dusollier, ‘The Master's Tools v. The Master's House: Creative Commons v. Copyright' (2006) 29 Columbia Journal of Law \& the Arts 3 271-293, 272
} 
Furthermore, it endeavours to lower the costs associated with copyright in terms of producing new works; the 'permissions process' for using copyrighted material can be cumbersome and expensive; therefore, $\mathrm{CC}$ has attempted to create a type of 'modularised' contract that creators can use to pre-authorise certain use(s) of their content17. The fulcrum of this position is the creator's control over content use through the $\mathrm{CC}$ licensing structure; conveying a formal expression of legal identity 18 which allows them to extract agreements on reproduction19. There are six licences to choose from and out of these, three permit commercial use (Attribution, Attribution-ShareAlike and Attribution-NoDerivs), whilst the remainder no not. However, it does not appear that the term 'commercial use' is defined, although it may be presumed to be the opposite of CC's definition of 'NonCommercial': 'primarily intended for or directed toward commercial advantage or monetary compensation'.20 There is acknowledgement that no activity may be completely separated from commerciality, but that the primary nature of the reuse is what should be considered 21 . It is important to note that all the non-commercial licences contain a special provision for

\footnotetext{
17 AK Goss, 'Codifying a Commons: Copyright, Copyleft, and the Creative Commons Project' (2007) 82 ChiKent L Rev 963-996, 963-964

${ }_{18}$ K Bowery, Law \& Internet Cultures (Cambridge University Press 2005) 169

19 WJ Gordon, 'An Inquiry into the Merits of Copyright: The Challenges of Consistency, Consent, and Encouragement Theory' (1989) 41 Stanford Law Review 1343-1460, 1418

${ }_{20}$ Creative Commons, 'Frequently Asked Questions' < https://creativecommons.org/faq/> accessed 1 November 2016

${ }_{21}$ Creative Commons, 'NonCommercial interpretation'

$<$ https://wiki.creativecommons.org/wiki/NonCommercial_interpretation> accessed 1 November 2016
} 
file-sharing (as this is deemed a commercial activity22), which is permitted, provided this is no monetary compensation 23.

The aim of these licences is (broadly) to authorise the use of copyrighted works for purposes that may otherwise constitute infringement under traditional copyright law24. All the licences also terminate automatically if a work is used contrary to the specified licence terms25. The component elements of the CC license 'kit', namely the 'Deed' and 'Licence Code' are intended to make the licence more accessible and understandable for users, as opposed to lawyers26 (on which particular emphasis was placed27). These are accompanied by an icon or graphic designed to indicate the key feature of the particular licence.

\section{Creative Commons and Copyright}

\footnotetext{
22 As the Court in Napster found, see A\&M Records Inc. (and others) v. Napster Inc., 239 F.3d 1004 (9 $9^{\text {th }}$ Cir. 2001), 912

${ }_{23}$ Creative Commons, 'Frequently Asked Questions' $<$ https://creativecommons.org/faq/> accessed 1 November 2016

24 S Dusollier, 'The Master's Tools v. The Master's House: Creative Commons v. Copyright' (2006) 29

Columbia Journal of Law \& the Arts 3 271-293, 271

${ }_{25}$ See the legal code s.6.a for all licences, available from $<$ http://creativecommons.org/licenses/ $>$ accessed 1

November 2016

26 B Bloemsaat and P Kleve, 'Creative Commons: A business model for products nobody wants to buy' (2009)

IRLCT 23(3) 237-249, 242

27 J Coates, 'Creative Commons - The Next Generation: Creative Commons licence use five years on' (2007)

SCRIPTed 4(1) 75
} 
The introduction of the $\mathrm{CC}$ system reflects the changes in the concentration and subsequent decentralisation of information (and content) production28 that digital technology facilitates 29. Nonetheless, the production and distribution of music in digital form still functions in accordance with the operation of rightsholders who have utilised copyright to preserve their pre-existing market operation. $\mathrm{CC}$ could also be seen as a response, not just to digital technology, but also to the effect it has had on copyright law30 which has been expansively applied by rightsholders to secure their rights in the digital environment.

Although CC has its own different rules31, copyright and CC can both operate jointly over music content and are therefore co-existent; copyright provides the underlying protection from which use can be administered via the $\mathrm{CC}$ licence. The fact that $\mathrm{CC}$ operates with copyright law as its regulatory underpinning is perhaps its most workable aspect as it does not require any restructuring of copyright law itself and is unlikely to prejudice the commercial interests served by the existing regime32. Nonetheless copyright is the inescapable legal interest that underpins the work itself.

\footnotetext{
28 Yochai Benkler, 'The Commons as a Neglected factor of Information Policy' (26th Annual Telecommunications Research Conference 1998) 24 <www.benkler.org/commons.pdf $>$ accessed 2 November 2016

29 L. Lessig, 'The Future of Ideas: The Fate of the Commons in a Connected World' (2002, Vintage Books), p8. 30 See generally, J Litman, 'Reforming Information Law in Copyright's Image' (1997) 22 U Dayton L Rev 587 619
}

31 LP Loren, 'Building a Reliable Semicommons of Creative Works: Enforcement of Creative Commons Licenses and Limited Abandonment of Copyright' (2007) 14 Geo Mason L Rev 271-328, 275 32 AK Goss, 'Codifying a Commons: Copyright, Copyleft, and the Creative Commons Project' (2007) 82 ChiKent L Rev 963-996, 992 
The CC licences seek to place such works in a 'commons' 33 ; a term that has come to be used increasingly over the last number of years to refer to wellsprings of creation that are outside of, or different from, the world of intellectual property34. In essence, the idea of a 'commons' refers to a situation where access to, and use of, a resource is organised on a nonexclusionary basis. Reduced to its conceptual minimum, it entails a situation where no specific individual or entity is recognised under the law as having a right to exclude others from access to and use of a given resource35. Nonetheless, it appreciates the realities of copybased digital reproduction and 'An information commons is possible because information is nonrival, and is an input and an output of its own production process'.36 Such a 'commons' could theoretically exist in the form of any available digital content, whether copyrighted or not. A great deal of infringement occurs every day37 and therefore the notion of a separate 'commons' does not apply well in the digital environment. Given the opportunities digital technology has afforded, the commons here can theoretically be any content in digital form provided that it is available or accessible to users, regardless of copyright protection38. It could be argued that because content is so easily available online, the boundaries between a

33 S Corbett, 'Creative Commons Licences, the Copyright Regime and the Online Community: Is there a Fatal Disconnect?’ (2011) MLR 74(4) 503-531, 518

34 J Boyle, 'Second Enclosure’ (2003) 66 Law \& Contemp Probs 33-74, 62

35 J Cahir, 'The withering away of property: the rise of the internet information commons' (2004) OJLS 24(4) $619-641,621$

36 Yochai Benkler, 'The Commons as a Neglected factor of Information Policy' (26th Annual Telecommunications Research Conference 1998) 21 <www.benkler.org/commons.pdf $>$ accessed 2 November 2016

37 R Polk Wagner, 'Information Wants to Be Free: Intellectual Property and the Mythologies of Control' (2003) Columbia Law review 103(4) 995-1034, 1010

38 D Hunter FG Lastowka, 'Amateur-To-Amateur' (2004) 46 William and Mary Law Review 951-1030, 985 
'commons' and protected content have blurred to the point of indivisibility as 'All popular music ... essentially, if not legally, exists in a public domain'.39 Indeed, copyrighted music has a central place in genres such as the practice of 'Plunderphonics' which is 'an umbrella term for any music made completely out of existing audio recordings, including copyrighted material, and then altered in some way to create a new composition'.40

Although the $\mathrm{CC}$ and copyright systems are co-existent insofar as $\mathrm{CC}$ is entirely dependent on copyright, copyright treats almost all unauthorised use of content as infringement; save for those which may be covered under the fair dealing exceptions, which themselves have been described in the past as 'rigid' 41 , or at least 'outdated' 42 . In comparison, $\mathrm{CC}$ pre-emptively delineates a sphere of use for the work according to the particular licence chosen. Furthermore, as digital consumption is copy-based, resource depletion is not an issue and in this instance, it could be argued that $\mathrm{CC}$ unintentionally creates a degree of artificial scarcity within the realm of available content by exercising licensing control to delineate a self-prescribed commons, albeit it one which available for legal use. This is because CC applies only to such self-prescribed works (as opposed to copyright's automatic protection) and as such, it fragments available content into a commons with different usage restrictions and enforces its own barriers based on its own licence terms. Therefore it can be argued that the effect of CC licencing is to define and re-establish boundaries within the volume of content accessible, and useable, to a creator.

\footnotetext{
39 J Oswald, 'Plunderphonics, or Audio Piracy as a Compositional Prerogative' (Plunderphonics, 1985) available from: <www.plunderphonics.com/xhtml/xplunder.html > accessed 1 November 2016 40 Ibid

41 Pro Sieben media AG v Carleton UK Television Limited and Another [1988) FSR 43 (HC) 48 42 I Hargreaves, Digital opportunity: a review of intellectual property and growth (Intellectual Property Office 2011), 40-43
} 
It is based on the premise of relocating power in the hands of the authors, but at the same time aims to be grounded in the expectations of users 43 (in terms of allowing the re-use of content) even though most $\mathrm{CC}$ users have played no part in the development of the licences44. As such, users of CC-licensed content are bound by one-way licence declaration on the part of the author and this has proved problematic in the past. Licensing mechanisms also exist under copyright law that allow pre-existing copyrighted content to be utilised in the creation of new music and arguably a CC licence attached to a work introduces another potential variable to be taken into account 45 over and above copyright and potential associated costs. There is also no guarantee that $\mathrm{CC}$-licensed content may be treated with any more (or less?) reverence than copyrighted content in respective the contexts of professional and amateur music production. This can be seen in the Freesound 46 project; a collaborative database of over fifty thousand $47 \mathrm{CC}$ licensed sounds48. Content on Freesound consists of samples, drum loops, and other electronically produced sounds available for others to use 49. Whilst it evidences the (worthwhile) ideological mind set of CC users who create music in

\footnotetext{
43 S Dusollier, 'The Master's Tools v. The Master's House: Creative Commons v. Copyright' (2006) 29

Columbia Journal of Law \& the Arts 3 271-293, 288
}

44 S Corbett, 'Creative Commons Licences, the Copyright Regime and the Online Community: Is there a Fatal Disconnect?’ (2011) MLR 74(4) 503-531, 505

45 B Bloemsaat and P Kleve, 'Creative Commons: A business model for products nobody wants to buy' (2009) IRLCT 23(3) 237-249, 246

$46<$ www.freesound.org $>$ accessed 1 November 2016

47 ccMixter, 'The Freesound project \& ccMixter' <http://ccmixter.org/freesound > accessed 1 November 2016

48 Freesound, 'About Freesound' <www.freesound.org/help/about/> accessed 1 November 2016

49 This has also been done commercially, with the dance producer Deadmau5 having released a sample CD-

ROM entitled 'XFER' (also the name of his record label) for producers. See Loopmasters, 'Deadmau5 XFER'

$<$ www.loopmasters.com/product/details/236> accessed 16 March 2016 
terms of creating content for use and re-use by others, $\mathrm{CC}$ does not take account of the differing attitudes individual creators may have towards these50. For example, one Freesound user, Nic Stage, uploaded a sample that came to be used by UK dance group the Prodigy on their 2009 album 'Invaders Must Die' (a UK number one which sold over 1 million units worldwide) and which was featured on their single 'Omen' 51 . The sample itself was subject to an Attribution licence and had no non-commercial restriction. However, problematically for Stage (and arguably more so for Creative Commons) he was not credited. Although the situation was ultimately resolved, Nic Stage had the option to sue for copyright infringement and/or a breach of the CC licence.

As already discussed, Copyright protection and the CC-licensing mechanism co-exist inasmuch that the sound recording receives automatic copyright protection upon fixation and the subsequent adoption of a CC-licence over it governing use/re-use. Although CC licences are deemed to be legally enforceables2, as a matter of practicality, the unauthorised use of the CC-licensed work (in this instance by the Prodigy) could be treated as an infringement of copyright and not a breach of the licence, although both can arise in the same instance. In addition, because a licence breach results in the termination of the $\mathrm{CC}$ licence itself, subsequent uses of the work are de facto copyright infringements. Therefore, CC's dependency on copyright means that in circumstances of alleged CC infringement, the

\footnotetext{
50 EE Johnson, 'The Economics and Sociality of Sharing Intellectual Property Rights' (2014) 94 Boston University Law Review 1935-1995, 1981

51 Nic Stage, 'Seems my sound was used without attribution...'<www.freesound.org/forum/legal-help-andattribution-questions/4189/> accessed 1 November 2016

52 Creative Commons, 'Frequently Asked Questions'

$<$ https://wiki.creativecommons.org/index.php/Frequently_Asked_Questions\#Are_Creative_Commons_licenses_ enforceable_in_a_court_of_law.3F $>$ accessed 7 November 2016
} 
perceived problems in copyright law that $\mathrm{CC}$ aims to overcome actually resurface. Although little of the associated CC case law53 deals with copyright infringement directly, it has been relevant to some of the decisions54. As mentioned above, the aims of the licences are to authorise the use of content where such use may otherwise infringe copyright law. Therefore, a breach of the licence necessarily entails a breach of copyright as well and this being the case, recourse to copyright law may be the inevitable option for the creator. Although CC appreciates the realities of the digital environment along with new and emerging forms of content use (and reuse), it ultimately fails to provide any alternative to copyright where use in contravention of the $\mathrm{CC}$ licence, i.e. unauthorised use, occurs. In this particular instance however, the remedy sought by Cage was the appropriate attribution for himself and/or Freesound as per the licence, following dialogue with the band's management. His further correspondence on the matter is also reflective of the CC ideology and the Freesound project in particular, and crucially is indicative of the mindset of those who have adopted the mechanism.

\section{Creative Commons and Intermediaries}

CC has had a strong uptake in terms of online content providers as well as facilitating the development of new and CC-focussed intermediaries that focus on CC-licensed content provision. Notwithstanding the issues discussed above, attention will now turn to these in order to assess their viability in facilitating a viable market for $\mathrm{CC}$ works. In contrast to the joint-operation of CC and copyright over content, this section will argue that when it comes to the exploitation of $\mathrm{CC}$ content, the relationship between the two actually operate to

\footnotetext{
53 'Case Law'<https://wiki.creativecommons.org/wiki/Category:Case_Law> accessed 6 November 2016 54 Curry v Audax 334492/KG 06-176 SR (District Court of Amsterdam, March 2006), Gerlach v DVU 16 O 458/10 (District Court of Berlin, October 2010) and, Avi Re'uveni v Mapa TA 3560/09, 3561/09 (Israel, 2011)
} 
segregate CC-licensed content in such a way so as to make a movement from 'amateur' to 'professional' content creation difficult.

As has been stated, CC aims to create an alternative market structure for creators participate in, as opposed to the 'traditional' (commercial) market engendered by copyright law and although $\mathrm{CC}$ also provides for non-commercial use, there has still been a substantial uptake of the licensing model. Flickr and Wikipedia are two large undertakings who employ CC licences, with one billion CC-licensed images on Flickr55 and Wikipedia having over 5 million articles in English56. Data.gov.uk, which although governed by Crown Copyright, has a significant $\mathrm{CC}$ endorsement as the content's licences are stated to be compatible with the Creative Commons Attribution License 4.057. However, when one looks more closely at the materials that are available on these (and other) sites, they function as a pool of content or perhaps more accurately 'resources' i.e. reference materials/information that can be accessed and used. This may explain why the sciences, libraries and academia have been most interested the licensing scheme58, as opposed to the content industries more widely. Despite this, $\mathrm{CC}$ can operate as an incentive to engage in creative practice as well as organise the way

\footnotetext{
55 'Explore/Creative Commons' <www.flickr.com/creativecommons/> accessed 2 November 2016

56 'Content Licensing' < http://en.wikipedia.org/wiki/Wikipedia>, and, 'English Wikipedia'

$<$ http://en.wikipedia.org/wiki/English_Wikipedia> accessed 6 November 2016. Wikipedia adopted CC

Attribution-ShareAlike licences in 2009. A full list of organisations that employ CC licences can be found on the 'Content Directories' page $<$ http://wiki.creativecommons.org/Content_Directories $>$ accessed 6 November 2016

57 Open Government Licence for public sector information <www.nationalarchives.gov.uk/doc/opengovernment-licence/version/3/> accessed 2 November 2016

${ }_{58}$ K Bowery, Law \& Internet Cultures (Cambridge University Press 2005) 167
} 
already-produced works are rationed and co-ordinated59; which in the online context is different from 'traditional' market-based content distribution governed by copyright. Indeed, the goals of CC with its focus on sharing and reuse are different from the profit-orientated approach of the music industry; such that the two should not necessarily be regarded as being in competition with each other (nor should it be assumed that $\mathrm{CC}$ is trying to overtake, or replace, existing mechanisms of content creation). Nonetheless, attention should be paid to outlets for such content in order to evaluate whether or not CC can provide an attractive alternative for creators.

As user-generated content proliferates, the traditional boundary between popular culture and amateur productions is changing with today's popular content sharing sites (such as YouTube) dominated by user-generated content 60 . CC has fostered its own online intermediaries and under many circumstances, sharing can be more beneficial than marketbased distribution61. Because they offer different possibilities from copyright, the CC licence options can act as a 'disintermediating' force because they enable end-to-end transactions of content. However they can also act as a 'reintermediating' force by allowing new services and communities to form around such content62. With the music industry focussing on fewer acts and taking on fewer risks63, there are opportunities for markets to develop and CC

\footnotetext{
59 WJ Gordon, 'An Inquiry into the Merits of Copyright: The Challenges of Consistency, Consent, and Encouragement Theory' (1989) 41 Stanford Law Review 1343-1460, 1393, and see generally 1388-1393 ${ }_{60}$ J Coates, 'Creative Commons - The Next Generation: Creative Commons licence use five years on’ (2007) SCRIPTed 4(1), 83

61 EE Johnson, 'The Economics and Sociality of Sharing Intellectual Property Rights' (2014) 94 Boston University Law Review 1935-1995, 1937 62 MW Carroll, 'Creative Commons and the New Intermediaries' (2006) Mich St L Rev 45-65, 47 63 A Harrison, Music: The Business (4th edn Virgin Books 2008) 49
} 
licences may therefore have the possibility of enabling new intermediaries64 to grow . One such intermediary is the Free Music Archive65 (FMA). A search on the FMA music website first and foremost reveals range of musical genres and sub genres66, many of which would arguably not be classed as 'mainstream'. Instead, these can be seen as 'niche' genres which although they may not have universal appeal in themselves, may combine to form a sizeable portion a music market when taken together. Another intermediary is Jamendo which provides a commercial outlet for CC-licenced music and one which is distinct from the copyright-based collecting agencies67. Specifically, its Jamendo Licensing arm allows for the commercial exploitation for music for those artists registered on it through a range of licences for both individuals (priced at £3.99) and companies/legal entitles (ranging from $£ 35$ to £209)68. Although artists using service are predominantly amateur, it appears that the prime motivator of both the amateur and professional artists is their adoption of the CC licences and

64 MW Carroll, 'Creative Commons and the New Intermediaries' (2006) Mich St L Rev 45-65, 49

65 Free Music Archive <http://freemusicarchive.org/> accessed 2 November 2016

${ }_{66}$ Specifically: Blues, Classical, Country, Electronic, Experimental, Folk, Hip-Hop, Instrumental, International, Jazz, Novelty, Old-Time/historic, Pop, Rock, Soul-R\&B and Spoken. Each of these have their own sub-genres of which there is not enough space to list fully. For example, the sub-genres of 'Rock' are: Garage, Surf, Goth, Indie-Rock, Industrial, Krautrock, Lo-Fi, Loud-Rock, Noise-Rock, Sludge, Metal, Black-Metal, Death-Metal, Grindcore, New Wave, Post-Rock, Space-Rock, Progressive, Psych-Rock, Punk, Electro-Punk, Hardcore, Thrash, No Wave, Post-Punk, Power Pop, Rock Opera and Shoegaze.

Genres $>$ Rock $<$ http://freemusicarchive.org/genre/Rock/> accessed 2 November 2016

${ }_{67}$ Frequently Asked Questions, 'What is free music?' <www.jamendo.com/faq > accessed 2 November 2016 ${ }_{68}$ Jamendo Licensing, 'Amazing music. Incredible prices'

$<$ https://licensing.jamendo.com/en/pricing?source=catalog_index $>$ accessed 2 November 2016. Jamendo also operate their own in-house credit system which may be purchased and used for various licensing bundles, see Jamendo Licensing, 'Credit packs' $<$ https://licensing.jamendo.com/en/credits?source=pricing_index $>$ accessed 2 November 2016 
its correspondence with the ethos of sharing69. This is perhaps indicative of the fact that audience-building and sharing-based distribution is seen as preferable in the current digital landscape and should not be underestimated. In such instances, CC can therefore enable the development untapped business models and markets70. Nonetheless, a transition from recognition to commercial exploitation would be problematic as 'Once an audience for an individual's work develops, the question of compensation becomes more fraught'.71

CC's underlying element of gratuity poses clear problem when compared to the preexisting revenue-based market structure which copyright supports. Recognised and centralised intermediaries are not necessarily the owners of the content, but through their market power they have been rendered 'in charge' of it. As such, they may be regarded as 'stewards' who provide access to, and streaming of, content to end-users and who are subsequently responsible for gleaning revenue for it. For example the 'Discover' feature on Spotify algorithmically tailors music suggestions to the user's 'taste', based on their listening habits72 and other commercial music intermediaries such as Tidal, Apple and Google offer similar services73. Such intermediaries can also operate in a more 'official' context as

${ }_{69}$ S Bazen, L Bouvard and JB Zimmerman, Musicians and the Creative Commons: A survey of artists on Jamendo’ (2015) Information Economics and Policy 32, 65-76, 69 and 74

70 J Coates, 'Creative Commons - The Next Generation: Creative Commons licence use five years on' (2007) SCRIPTed 4(1), 85

71 D Zimmermanm, ‘Authorship Without Ownership: Reconsidering Incentives in a Digital Age’ (2003) DePaul Law Review 1121-1170, 1145

72 Spotify, 'Discover' < https://support.spotify.com/uk/using_spotify/discover_music/discover/> accessed 2 November 2016

73 Ben Popper, 'Tastemaker: How Spotify's Discover Weekly cracked human curation at internet scale’ (The Verge, 30 September 2015) < www.theverge.com/2015/9/30/9416579/spotify-discover-weekly-online-musiccuration-interview $>$ accessed 2 November 2016 
aggregators and measurers of commercial success through the official charts system 74 . Streamed music has a role here too and is combined with physical sales and downloads to gauge a song's popularity75. However, this supports a much more revenue-orientated model of eligibility requiring a minimum pricing threshold; for example, digital tracks must have a minimum price of $£ 0.40$ GBP as 'singles' in order to be eligible 76 . Similarly, one hundred streams of a song is equated to one download thereof77. A revenue-based model is arguably necessary as music production can involve high initial costs78 and it can take up to 1 million GBP to launch a new music act79. This has traditionally been mediated through capital 80 ; implicating a broader economic structure at an 'industrial' level and which copyright provides for the music industry. $\mathrm{CC}$ therefore assumes a limited view of creators as being those who may always permit the use/re-use of their work without potential remuneration; 'This ethos of sharing suggests that the economic model put in place by the Creative Commons licenses is one of gratuity'.81 Although it needs to be recognised that market

\footnotetext{
74 For example in the UK, see Official Charts <www.theofficialcharts.com> accessed 2 November 2016 75`Rules for Chart Eligibility Singles’ (Official Charts Company, May 2016) 7 $<$ www.officialcharts.com/media/650606/official-uk-charts-singles-chart-rules-may-2016.pdf $>$ accessed 6 November 2016

76 ibid, 4-5

77 ibid, 7

78 AK Goss, 'Codifying a Commons: Copyright, Copyleft, and the Creative Commons Project' (2007) 82 ChiKent L Rev 963-996, 974

79 A Harrison, Music: The Business (4th edn Virgin Books 2008) 285

80 D Hunter and FG Lastowka, ‘Amateur-To-Amateur' (2004) 46 William and Mary Law Review 951-1030, 979

81 S Dusollier, 'The Master's Tools v. The Master's House: Creative Commons v. Copyright' (2006) 29

Columbia Journal of Law \& the Arts 3 271-293, 281
} 
transactions are not efficient except for a minority of works with sufficiently high commercial value and that money is not an ideal motivator for creativity and innovations2, it can also be argued that very few artists utilising CC licensing will be able to sustain a living purely from their music83. As a result, it is questionable whether creators would then have sufficient incentives to produce new works in a system is based on freedom and sharing.

Owing to the joint operation of copyright and a $\mathrm{CC}$ licence over a piece of content, it theoretically means that there is nothing preventing the creator from exploiting their work (through copyright) outside of the CC system as the two are not mutually exclusive. However, should content be made freely available under CC, then direct commercial exploitation of the content through copyright becomes practically impossible; for example, a record label would be unlikely to be willing to licence and exploit something which is (or has been) already available online for free. Alternative exploitation may be an option which may be realised through so-called '360 deals' which, broadly speaking, give the rightsholder (i.e. the record label) a share of merchandising, ticket sales and many important aspects of an artist's career84.

$\mathrm{CC}$ is not necessarily a mechanism to facilitate profit directly from content, other than the 'value' that comes from attribution. This serves as an important contrast to the 'traditional' operation of the music industry which places the utmost importance on the

\footnotetext{
82 EE Johnson, 'The Economics and Sociality of Sharing Intellectual Property Rights' (2014) 94 Boston University Law Review 1935-1995, 1994

83 S Bazen, L Bouvard and JB Zimmerman, 'Musicians and the Creative Commons: A survey of artists on Jamendo’ (2015) Information Economics and Policy 32 65-76, 76
}

84 A Harrison, Music: The Business (4th edn Virgin Books 2008) 67-69 
market and may arguably be modified to take account of their own interests85 (for example, the inclusion of music streaming in the charts, as mentioned above). Attribution may be the most important thing above all else86 for creators as was the case with Nic Stage discussed above; in which case CC may be of benefit because 'Copyright's processes are relevant primarily to centralized copyright industries ... For amateurs, however, it isn't clear that copyright law is very important at all'. 87 However, such an 'amateur' context may be to the movement's detriment as creators would be unlikely to devote themselves fully to creative practice if they cannot profit from the value that others place in their work88. It needs to be borne in mind that although CC may make it difficult for creators to profit from their endeavours, this is not the goal of the movement; arguably, the real benefit of $\mathrm{CC}$ will be for creators who now have the ability to exploit their own works without, hopefully, being exploited themselves 89 .

$\mathrm{CC}$ is inherently dependent on copyright in order for the licences to function and although it seeks to overcome the costs and burdens copyright may create when it comes to content production, there are still clear problems CC faces. Although there are mechanisms to facilitate audience-building, the only mechanism by which to receive 'compensation' is the

\footnotetext{
85 S Dusollier, 'The Master's Tools v. The Master's House: Creative Commons v. Copyright' (2006) 29

Columbia Journal of Law \& the Arts 3 271-293, 292-293

86 P Flichy, 'Discourse on the New Economy - passing fad or mobilizing ideology?' in E Brousseau and N Curien (eds), Internet and Digital Economics: Principles, Methods and Applications (Cambridge University Press 2008)

87 D Hunter and FG Lastowka, 'Amateur-To-Amateur' (2004) 46 William and Mary Law Review 951-1030, 1026

88 ibid, 1137

89 M Fox, T Ciro and N Duncan, 'Creative Commons: an alternative, web-based copyright system' (2005) Ent LR (2005) 16(5) 111-116, 116
} 
pre-existing copyright market structure which $\mathrm{CC}$ is unable to incorporate itself in; leaving it vulnerable to misuse (including misuse by professionals). This makes it difficult to emerge from its niche status despite the availability of CC-focussed intermediaries. In contrast with the dual relationship copyright and $\mathrm{CC}$ has over content, at this juncture there is an insurmountable segregation between content protected by copyright and content specifically governed by a $\mathrm{CC}$ licence. $\mathrm{CC}$ artificially constrains exploitation of the $\mathrm{CC}$ content through its own associated intermediaries; making both the creators and the intermediaries unable to engage with the copyright-driven commercial structure that is already in place. This is perhaps a fundamental problem for the CC strategy; the fact that it operates primarily a 'free' basis suggests that a market could not develop for such content. Although there are mechanisms to facilitate audience-building; the only mechanism by which to receive 'compensation' is the pre-existing copyright structure which may be preferable 90 as it can allow for increased exploitation which can subsequently increase value. However, it does provide valuable recognition of the value of 'free' and the non-financial value, or recognition, that corresponds with this via attribution.

\section{Conclusion}

The CC strategy is not as revolutionary at it may perhaps first sound because it still effectively operates on the basis of copyright law, but this does not mean that the movement has no value. It has the benefit of being designed with the realities of digital production and distribution in mind and can be considered a more proactive response to the problems of digital copyright law than the mere updating of copyright law. Although CC may have

\footnotetext{
90 WJ Gordon, 'An Inquiry into the Merits of Copyright: The Challenges of Consistency, Consent, and Encouragement Theory' (1989) 41 Stanford Law Review 1343-1460, 1419
} 
benefit in addressing user-norms engendered by digital technology, that is not to say it will provide a viable solution on its own. $\mathrm{CC}$ resources and copyrighted resources are indistinct in their practical availability and by artificially aiming to create 'scarcity' through its licensing system, CC limits users (and itself) to a relatively narrow and niche body of content. Theoretically, CC-licensed content is only available to serve as inputs for creative works. As such, the self-imposed architecture of CC artificially narrows the available creative resources to other CC-licensed works. Furthermore, it is questionable whether a viable market structure can develop in light of this because of the stipulated noncommercial/free and predominantly amateur context in which it operates. However, this does not jeopardise the movement's validity as an alternative to the traditional copyright market structures and for many (as is the case with Jamendo, mentioned above), could be seen as its key feature.

The CC movement recognises the opportunities afforded by digital technology for creation and dissemination of content. It has also afforded the development of CC-themed digital intermediaries to facilitate the distribution of content. Nonetheless, one must take a practical approach when looking at the markets for CC-licensed work. Many of the large organisations that utilise the licensing system are not necessarily content distribution services, but 'reference' services. As such, there is little (if any) revenue-generating market for such content. It may also be suggested that artists adopting $\mathrm{CC}$ licensing, whilst making a noble statement against the constraints of copyright law, are missing the point. The pure existence of such an enterprise demonstrates the potential scale and niche value the Internet is able to provide. The Internet is able to sustain a virtually infinite demand curve and while this may 
plateau towards the bottom of the arc, it may extend as far as there is content to support it91 and copyright law can enable its exploitation.

CC may facilitate the growth of intermediaries providing licensed content as (financial) copyright licensing costs would not be incurred. This is no bad thing as providing an additional and alternative means of content dissemination92. However, copyright and commerciality still have primacy in this respect regarding the music market. It should be borne in mind that copyright also affords the option for gratuity; there is precedent in the music industry for the assumption that 'giving something away' can be beneficial, at least in terms of building a reputation for the artist93. In this context, the controlling legalities of copyright can be forgone (and in some instances, actively discarded by the artist); and in such circumstances, a CC licence is unlikely to make much of a difference. Furthermore, 'attribution' is a necessary component in terms of building an audience, and this would still be the case without an attached CC licence. Successful content exploitation it ultimately depends on building mutually beneficial relationships94 between artists and audience, which can be done just as easily through copyright and on the part of initiatives by the artists/creators themselves in presenting content to users and without such a formalised mechanism as CC licensing. Digital technology also facilitates this further.

\footnotetext{
91 See generally C Anderson, The Longer Long Tail (Updated and Expanded Edition), (Random House 2009) 92 D Hunter and FG Lastowka, 'Amateur-To-Amateur' (2004) 46 William and Mary Law Review 951-1030, 1018

93 See the examples of Prince, Wilco, OK, Radiohead (in a way), and Trent Reznor in G Kot, Ripped: How the Wired Generation Revolutionised Music' (Scribner 2009), chapters 5, 8, 19 and 20 94 MF Schultz, 'Fear and Norms and Rock \& Roll: What Jambands Can Teach Us About Persuading People to Obey Copyright Law’ (2006) 21 Berkeley Technology Law Journal 651-728, 657
} 
Whilst it may be concluded that the CC licensing system is flawed, that is not to say it has no benefit whatsoever. The dissemination of content under CC licences may be effective in providing creators with new opportunities95 (individually or via an intermediary) built on the value of attribution, although care should be exercised so as not to engender a 'gift culture'96. However, it is argued that $\mathrm{CC}$ existence as a practical, but also political97 movement is its strongest virtue. Copyright law is, and has been, subject to the lobbying interests of the content industries98; therefore, CC's a symbolic value based around the philosophies of sharing and contribution operates as an important counter-point to the aggressive and restrictive strategies employed by the music industry.

${ }_{95}$ S Dusollier, 'The Master's Tools v. The Master's House: Creative Commons v. Copyright' (2006) 29 Columbia Journal of Law \& the Arts $3271-293,281$ 96 AK Goss, 'Codifying a Commons: Copyright, Copyleft, and the Creative Commons Project' (2007) 82 ChiKent L Rev 963-996, 995

${ }_{97}$ S Dusollier, 'The Master's Tools v. The Master's House: Creative Commons v. Copyright' (2006) 29 Columbia Journal of Law \& the Arts 3 271-293, 273

${ }_{98}$ K Bowery, Law \& Internet Cultures (Cambridge University Press 2005) 108 